\title{
On Colourings of Wheel Graph (Wn)
}

N. Ramya*

Dept. of Mathematics, Bharath University, Selaiyur, Chennai, India; nramyas@yahoo.com

\begin{abstract}
We present a unique way of coloring, star coloring and acyclic coloring of wheel graph $W n, n \geq 4$.
\end{abstract}

Keywords: Acyclic Coloring, Coloring, Star Coloring, Wheel Graph (Wn)

\section{Introduction}

Graph coloring is a special case of graph labelling. It is an assignment of labels traditionally called 'colors' to elements of a graph subject to certain constraints. In its simplest form, it is a way of coloring the vertices of a graph such that no two adjacent vertices share the same color. The first result about graph coloring deal almost exclusively with planal graph in the form of the coloring of maps.while trying to color a map of the countries of England, Guthrie ${ }^{1}$ postulated the four color conjecture, nothing that four colors were sufficient to color the map so that no regions sharing a common border receives the map so that no regions sharing a common border receives the same color. The same problem was presented earlier by August Mobins ${ }^{2}$

The smallest number of colors needed to color a graph $\mathrm{G}$ is called its Chromatic number, $\Psi(\mathrm{G})$. In a graph $\mathrm{G}$ any path of length three is not bicolored is called star coloring of $\mathrm{G}$. In a graph $\mathrm{G}$, any cycle in $\mathrm{G}$ is not bicolored is called acyclic coloring ${ }^{3,4}$.

\section{Main Results}

\subsection{Result 1}

A wheel graph Wn $n \geq 4$ has chromatic number is 4 when $\mathrm{n}$ is even. A cyclic chromatic number of this graph also 4 and star chromatic number is 4 .



Coloring has given by the following steps:

1) Central vertex has given always 1

2) Outer vertex from $v_{2}$ to $v_{2 n-1}$

Coloring has given periodically

If $\mathrm{v}_{2}$ has coloring 2 then $\mathrm{v}_{3}$ has coloring 3

Similarity for $\mathrm{v}_{4}, \mathrm{v}_{5}$

The last vertex $\mathrm{v}_{2 \mathrm{n}}$ has to be given 4

Acyclic coloring can be given as follows

1) For central vertex coloring has been given 1 .

2) For v2, v3, v4 coloring has to be given 2, 3, 4 respectively.

In general $\mathrm{v}_{2 \mathrm{n}-2}, \mathrm{v}_{2 \mathrm{n}-1}, \mathrm{v}_{2 \mathrm{n}}$ coloring has to be given periodically $2,3,4$

Eg W10: 


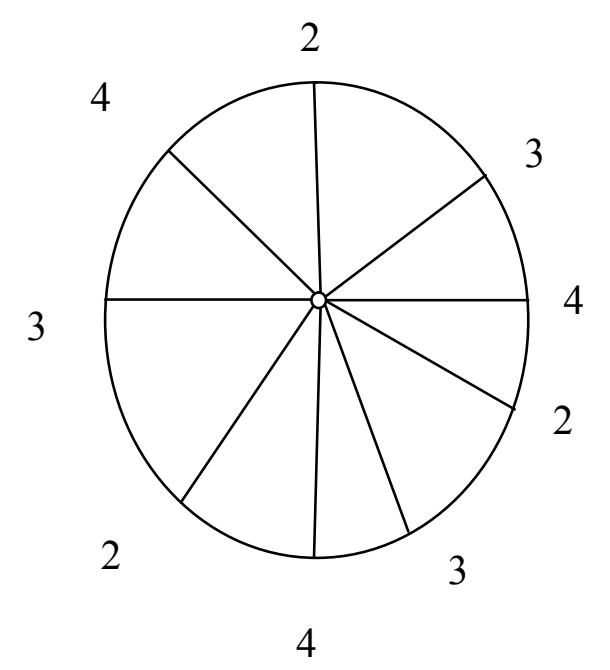

\subsection{Result 2}

A wheel graph Wn $\mathrm{n} \geq 4$ has chromatic number is 3 . When $\mathrm{n}$ is odd. A cyclic chromatic number and star chromatic number of these graphs are same as result 1.
Here $\mathrm{v}_{1}$ has 1 and $\mathrm{v}_{2}, \mathrm{v}_{3}$ has coloring 2,3 respectively similarly other for all vertices, coloring has to be done periodically.

\section{Conclusion}

Thus we conclude that in this wheel graphs having same coloring. i.e. $\Psi(\mathrm{G})=\Psi \mathrm{s}(\mathrm{G})=\Psi \mathrm{a}(\mathrm{G})$. It is of interest to look in to these kind of graphs of harmonious coloring, b-coloring, grundy coloring etc.

\section{References}

1. Guthrie F. Note on the coloring of Maps. Proc Roy Soc. Edinburgh 10. 1878-1880; 727-28.

2. Mobius F. The four color problem. Wikipedia; 1840.

3. Fertin G, Raspaud A, Reed B. Star coloring of graphs. J Graph Theory. 2004; 47(3):163-82.

4. Lyons A. Acyclic and star colourings of joins of graphs and an algorithm for cographs CTW. 2009; 199-202.

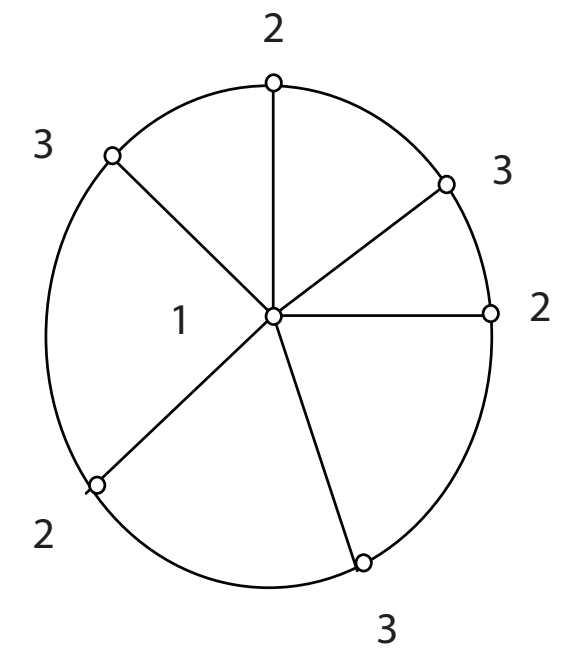

